Estrategias de intervención

\title{
PREVENCIÓN DE QUEMADURAS INFANTILES EN TIEMPOS DE PANDEMIA: UNA CAMPAÑA VIRTUAL E INTERACTIVA
}

\section{CHILDREN BURN INJURY PREVENTION IN PANDEMIC TIMES: A VIRTUAL INTERACTIVE CAMPAIGN}

\section{Carmina Domic Cárdenas}

Enfermera Matrona, Magister en Enfermería

Corporación de Ayuda al Niño Quemado, Dirección de Extensión, Docencia e Investigación, Subdirectora de Docencia y Extensión. Santiago, Chile cdomic@coaniquem.org https://orcid.org/0000-0001-8224-8293

\section{Carola Paredes Mayor}

Enfermera con diploma académico en Enfermería Comunitaria

Corporación de Ayuda al Niño Quemado, Dirección de Extensión, Docencia e Investigación, Enfermera de Extensión, Santiago, Chile

carolaparedes@coaniquem.org

https://orcid.org/0000-0001-9643-0467

Artículo recibido el 23 de marzo de 2021. Aceptado en versión corregida el 28 de julio de 2021.

\section{RESUMEN}

Las quemaduras infantiles pertenecen al grupo de los traumatismos y son lesiones que pueden producir la muerte o dejar secuelas graves permanentes. Son eventos que se producen con mayor frecuencia en niños menores de cinco años y ocurren dentro de su hogar. La mayoría de estos eventos se pueden prevenir con actividades de educación a la comunidad y campañas masivas. En marzo del 2020 se suspenden las actividades escolares presenciales en Chile por la pandemia de Coronavirus, lo que obliga a la permanencia de los niños en sus hogares. Esto se transforma en un riesgo de aumento de quemaduras, por lo que se diseña una campaña virtual e interactiva, dirigida a niños pre-escolares y escolares de primer ciclo, creando videos específicos para diferentes agentes causales de quemaduras con personajes animados y que finalizan con una invitación a enviar un dibujo de lo aprendido en el video. Con los dibujos recibidos se crea un nuevo video para compartir en redes sociales. Los niños logran plasmar en sus dibujos los mensajes educativos que han recibido y en cinco meses de campañas, con once videos distintos, se logra recibir 2.166 dibujos de niños chilenos y 317 dibujos del extranjero. La campaña se publica y difunde por redes sociales, considerándose como un medio útil y eficiente para entregar contenidos educativos en salud a la comunidad.

Palabras clave: Quemaduras, prevención primaria, educación en salud, pediatría. 


\section{ABSTRACT}

Pediatric burn injuries are a type of trauma that can lead to death or permanent scarring. There is a higher frequency of these events inside the home, mainly amongst children under five. Educational activities in the community and mass prevention campaigns help prevent most of these occurrences. In March 2020, due to the Coronavirus pandemic, the suspension of in-person classes and school activities forced children to stay inside their homes, increasing the risk of burn-related injuries. A virtual and interactive campaign addressed to pre-and elementary-school level children was created, with eleven videos, each specific to different burn agents, using animated characters. At the end of every video, there was an invitation to draw what they learned. With the received drawings a response video was created and shared on social media. Through their drawings, children managed to convey the educational message they received. During five months of the campaign, we received a total of 2,166 drawings from Chilean children and 317 from children from other countries. The campaign was publicized on social media because of its usefulness and efficiency to deliver educational content about community health.

Keywords: Burns, health promotion, health education, pediatrics.

\section{http://dx.doi.org/10.7764/Horiz_Enferm.32.2.212-222}

\section{INTRODUCCIÓN}

Las quemaduras son lesiones producidas en los tejidos vivos, debido a la acción de diversos agentes físicos: llamas, líquidos u objetos, radiación, corriente eléctrica, frío; químicos (cáusticos) y biológicos. Pueden provocar daños que van desde un simple eritema transitorio en la piel, hasta la destrucción total de las estructuras $^{(1)}$.

En el mundo, los traumatismos se encuentran entre las principales causas de muerte y discapacidad permanente entre los niños de 5 a 14 años, categoría en la que se incluyen las quemaduras ${ }^{(2)}$.

En lo que se refiere a mortalidad y población, se sabe que la tasa de mortalidad en pediatría por quemaduras en países de ingresos bajos y medios es 11 veces mayor que la de países de altos ingresos (4,3 por 100.000 vs. 0,4 por 100.000). Al mismo tiempo, los preescola- res (niños menores de 5 años) son quienes tienen la tasa de mortalidad más alta ${ }^{(3)}$. Las lesiones asociadas a riesgos ambientales y características familiares, son la primera causa de mortalidad, discapacidad y uso de servicios de salud en Estados Unidos para niños y adolescentes entre 1 y 19 años de $\operatorname{edad}^{(4)}$.

Tanto en la etapa aguda como en la rehabilitación posterior, las quemaduras provocan un gran sufrimiento al afectado y a su familia, importantes gastos en rehabilitación y, en muchos casos, daños emocionales, sociales, de crecimiento, desarrollo y estéticos que los acompañan durante toda la vida ${ }^{(1)}$. La carga económica asociada a las quemaduras infantiles incluye costos médicos a corto y largo plazo, con pérdida de productividad ${ }^{(4)}$. Los daños físicos derivados de las quemaduras representan la tercera causa de hospital- 
lización y muerte por trauma en los niños chilenos. La mayor frecuencia de quemaduras ocurre en el grupo de menores de 5 años, situación similar a la registrada en estudios hospitalarios de otros países ${ }^{(5)}$, y se ha demostrado que la mayoría de las quemaduras en niños ocurren en el $\operatorname{hogar}^{(5,6)}$.

En cuanto a los factores de riesgo, las quemaduras están fuertemente asociadas a pobreza, baja alfabetización dentro de la familia, hacinamiento, edad, supervisión de niños, riesgos ambientales, medidas de seguridad no adecuadas para uso de gas y electricidad, entre otros ${ }^{(3,4,6)}$.

La Organización Mundial de la Salud (OMS) califica a las quemaduras infantiles como "traumatismos involuntarios" dado que la mayoría no son producto de la intencionalidad; tienen la posibilidad de ser evitados y se conoce la causa aparente, por lo que no se denominan ni son considerados como accidentes $^{(7)}$. Esto motiva la incorporación de la prevención de quemaduras infantiles en acciones de promoción de la salud.

En base a la definición establecida en la Conferencia Internacional de Ottawa en 1986, la promoción de la salud es "el proceso de capacitar a las personas y a las comunidades para que aumenten el control sobre los determinantes de la salud, y, por lo tanto, mejoren su salud"(8).

La Promoción de la Salud requiere de la participación, inclusión e implicación de distintos actores sociales, en el ámbito de lo individual y familiar, y entornos más cercanos. Esto para incidir en cambios culturales más profundos que, permita a las personas ser protagonistas de sus propios procesos de cambio ${ }^{(9)}$.
En Chile, las acciones del Programa de Promoción de la Salud se enmarcan en la Estrategia Nacional de Salud, que en su objetivo estratégico $\mathrm{N}^{\mathrm{o}} 3$ propone la meta de Salud Óptima: "Desarrollar hábitos y estilos de vida saludables, y que favorezcan la reducción de los factores de riesgo asociados a la carga de enfermedad de la población, tales como el consumo de tabaco, consumo de alcohol, sobrepeso y obesidad, sedentarismo, conducta sexual y consumo de drogas ilícitas"(9). Un plan de prevención efectivo debe ser multisectorial e incluir esfuerzos para: aumentar conciencia del problema, desarrollar e implementar políticas efectivas, describir la carga e identificar factores de riesgo, definir prioridades de investigación con promoción de intervenciones prometedoras y proveer programas de prevención de quemaduras, entre otras ${ }^{(6)}$.

En numerosos países se ha comprobado que las intervenciones prevéntivas en los sectores de la salud y la gestión ambiental son eficaces para proteger a los niños de la exposición a factores peligro$\operatorname{sos}^{(7)}$. Chile es un país que se destaca por la realización de varias iniciativas que buscan promover la salud en su población, incluyendo a niños y niñas en edad preescolar, con la participación de diversas entidades ${ }^{(10)}$.

Mistry $^{(11)}$ señala cuatro pilares fundamentales de la salud en la infancia: cuidado receptivo, entornos seguros y protegidos, nutrición adecuada y apropiada, y comportamientos que promueven la salud; tres de los cuales son primordiales para evitar quemaduras si enfocamos nuestras acciones a ellos. Desde el punto de vista evolutivo y madurativo del niño, 
Prevención de quemaduras infantiles en tiempos de pandemia...

las edades tempranas son consideradas como una etapa clave en la integración de hábitos saludables, así como en el aprendizaje de prácticas beneficiosas para la salud que pueden perdurar a lo largo de la vida ${ }^{(8)}$. La interacción entre experiencias, ambientes y biología son influencias poderosas en salud infantil y ejercen sus efectos hasta la adolescencia o incluso la adultez ${ }^{(11)}$. Por su parte, el ámbito escolar es un espacio primordial para el desarrollo de acciones orientadas a promover la salud y lograr el desarrollo integral, construyendo ambientes saludables e inclusi$\operatorname{vos}^{(10)}$.

Existen múltiples mecanismos para que la comunidad pueda acceder a educación en salud y diferentes herramientas para ello, dependiendo de la población objetivo. No se utiliza el mismo material para adultos que para adolescentes o niños. Varía la forma de entregar los contenidos, el lenguaje, el canal, la metodología y otras características. En el mundo actual se necesita maximizar el acceso de la población a la educación en salud, a través de mejores estrategias e incorporando el uso de tecnologías digitales.

Las tecnologías de la información y comunicación se han convertido en una potencial fuente de promoción de la salud, en particular en pediatría. Cada vez más personas buscan en Internet información sobre salud. También, cada vez más, los profesionales de la salud se apoyan en esta modalidad como una herramienta de trabajo $^{(12)}$.

Para diseñar una campaña de prevención de quemaduras dirigida a niños se debe considerar las tecnologías digitales con las que los nativos digitales se comunican en su vida diaria y utilizarlas para comunicar el conocimiento del contenido pedagógico de una manera más atractiva $^{(13)}$. Los teóricos de la educación, ya sean conductistas, cognitivistas o constructivistas, coinciden en que una forma efectiva de facilitar aprendizaje es brindar a los alumnos la oportunidad de participar activamente en actividades y procesos que son significativos para ellos $^{(13)}$. Si se busca la participación activa de niños y niñas escolares y preescolares en una campaña de prevención podemos identificar un punto común para todos, como lo es la expresión a través del dibujo. Por medio del dibujo pueden incorporar los conceptos aprendidos y participar activamente; al compartir sus dibujos se genera un proceso interactivo, dinámico y que tiene el potencial de generar nuevos aportes a la campaña.

La educación artística busca desarrollar seres íntegros y aporta los elementos necesarios para que, considerando la edad y el grado de discernimiento de cada niño, fortifique su personalidad y su correspondencia con el entorno natural y social ${ }^{(13)}$. El dibujo es una construcción individual y cognoscitiva, cuyo aprendízaje no está subordinado a las normas estrictas a las que se ven sometidos el lenguaje $\mathrm{u}$ otros sistemas de comunicación, como pueden ser la matemática o la lógica ${ }^{(14)}$. La importancia del dibujo radica en que, para poder realizarlo, el niño debe, ante todo, interpretar la realidad, ya que para configurar una interpretación se requiere cierto grado de imaginación que supone comprender la realidad, sin la cual es imposible entender el mundo que nos rodea. Es a través del dibujo como el niño manifiesta el desarrollo de su pensamiento ${ }^{(14)}$. 
La educación preescolar en Chile ha dictado un listado único de objetivos mínimos que se encuentran en las "Bases Curriculares". Las referentes a la Educación Parvularia del año 2018 en el "núcleo de identidad y autonomía" y en el "núcleo de convivencia y ciudadanía" incorporan el uso de las tecnologías de la información y comunicación (TICs) para que los niños puedan reconocer y comunicar emociones, anticipar acciones y prever situaciones o desafíos, manifestar empatía y solidaridad, participando de acciones de escucha, apoyo y colaboración ${ }^{(15)}$.

Contemplando los aspectos mencionados, se respalda la creación de una campaña preventiva en el marco de la promoción de la salud, que esté orientada a disminuir las quemaduras infantiles, considerando las consecuencias personales, familiares, económicas y sociales que implican. La posibilidad de que los niños puedan participar en la campaña incorporando los contenidos a través de la realización de dibujos puede ser un factor favorable para el éxito de una campaña.

\section{ESTRATEGIA DE INTERVENCIÓN}

Durante los últimos 30 años la Corporación de Ayuda al Niño Quemado (COANIQUEM) ha sido líder en el desarrollo y ejecución de campañas de prevención de quemaduras infantiles en Chile. El inicio de la pandemia por COVID-19 en marzo de 2020 generó la interrupción de actividades escolares presenciales y la permanencia de los niños en sus hogares por un tiempo indefinido. Dado que las quemaduras infantiles se producen preferentemente dentro del hogar, COANIQUEM propuso crear una campaña virtual para educar a los niños sobre los riesgos de quemaduras y así enseñarles los agentes causales y mecanismos más comunes por los que se producen estas lesiones. Es así como se crea la "Campaña internacional, virtual e interactiva de prevención de quemaduras con Santi" dirigida a niños menores de 12 años. Entre los recursos para trabajo de prevención, COANIQUEM, con el aporte del Art Center College of Design de Pasadena, California, ha desarrollado tres personajes infantiles: Santi (mascota felina, traviesa y activa), Camila y Lucas (hermanos que cuidan a la mascota Santi). Estos personajes fueron creados específicamente para enseñar a los niños los riesgos de quemaduras, representados por las acciones que la mascota Santi realiza al acercarse a los agentes causales que producen quemaduras, y sus amigos Camila y Lucas que están siempre advirtiéndole a tiempo del peligro. De esta forma se cambia la manera tradicional de presentar el riesgo; la que era representada por un niño en la situación de quemarse, con la posibilidad de que la acción fuera imitada por los niños que la vieran. Además, se apela al cariño y cuidado que tienen los niños por sus mascotas y se les da un rol protagónico positivo.

Para el desarrollo de la campaña se realizaron 11 videos animados de los personajes, cada uno de aproximadamente 90 segundos de duración y que abordan distintos agentes causales que producen quemaduras. Los videos finalizan con una invitación a que los niños dibujen lo que aprendieron en el video y envíen una foto del dibujo a COANIQUEM. La cantidad de dibujos recibidos en respuesta será considerada un indicador de medición para la efectividad de la campaña. La campaña 
Prevención de quemaduras infantiles en tiempos de pandemia...

se ejecutó en el periodo comprendido entre el de 30 de junio y el 24 de noviembre 2020 .

Se recibieron dibujos de niños con edades comprendidas entre los 2 años y los 15 años, superando el rango etario al cual estaba dirigido el material (menores de 12 años de edad). Cada dibujo debía expresar lo que el niño aprendió al ver el video, cada uno de ellos fue revisado identificando que incorporara aspectos relacionados a la campaña: personajes del video y contenido de agente causal de quemaduras mencionado en el video. En muchos casos se identificó además cómo el niño incorpora en el dibujo a su propia mascota o reemplaza el modelo del agente casual del video por el que posee en su casa (ejemplo: estufa a gas que aparece en video y el niño dibuja estufa a leña, o reemplaza a Santi por su propio gato gris) (Figuras 1 y 2).

Esto confirma el proceso mental que realiza el niño de interpretar la realidad, aplicar un proceso creativo y traducirla en su propio lenguaje gráfico. Tal como indica la evidencia, cuando el niño crea un dibujo, necesariamente realiza una interpretación y comprensión de la realidad que lo lleva a entender el mundo que lo rodea (Figuras 3 y 4 ).

Figura 1. Dibujo en que el niño reemplaza el modelo del agente casual del video por el que posee en su casa.

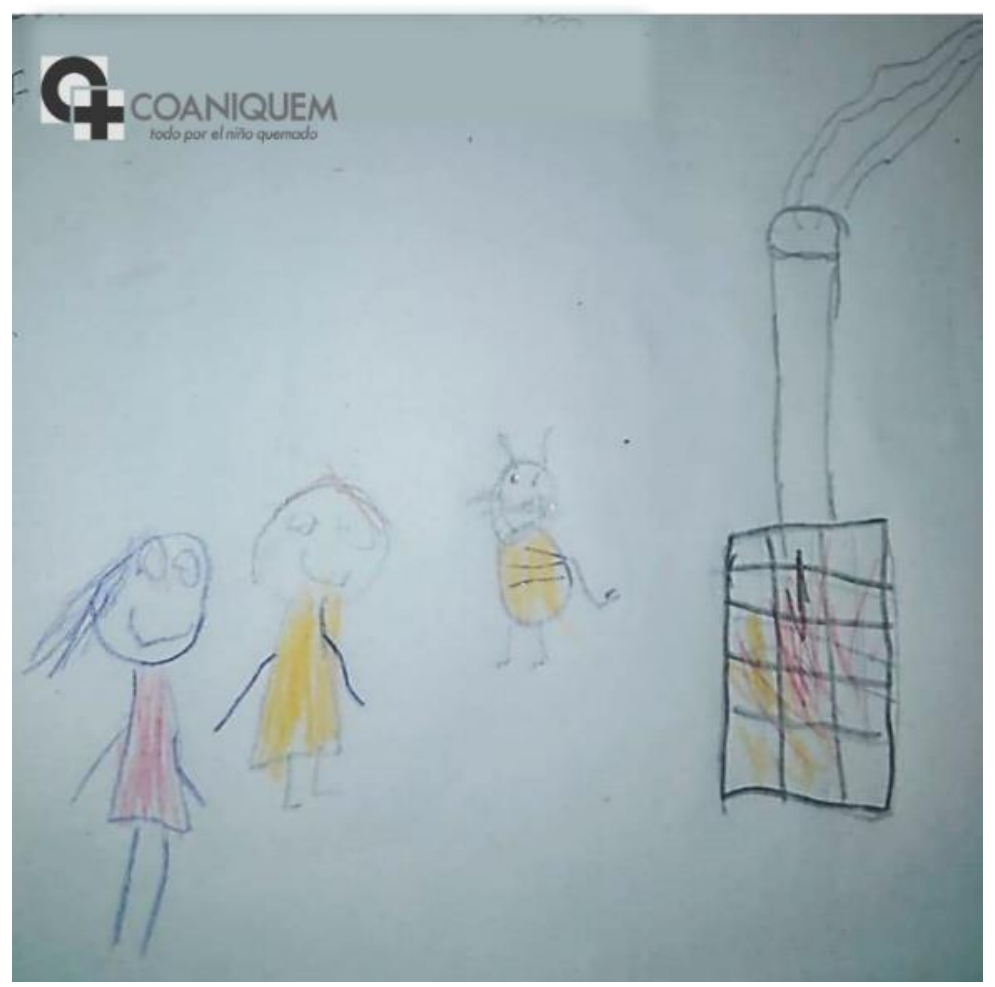


Figura 2. Dibujo en que el niño incorpora a su propia mascota.

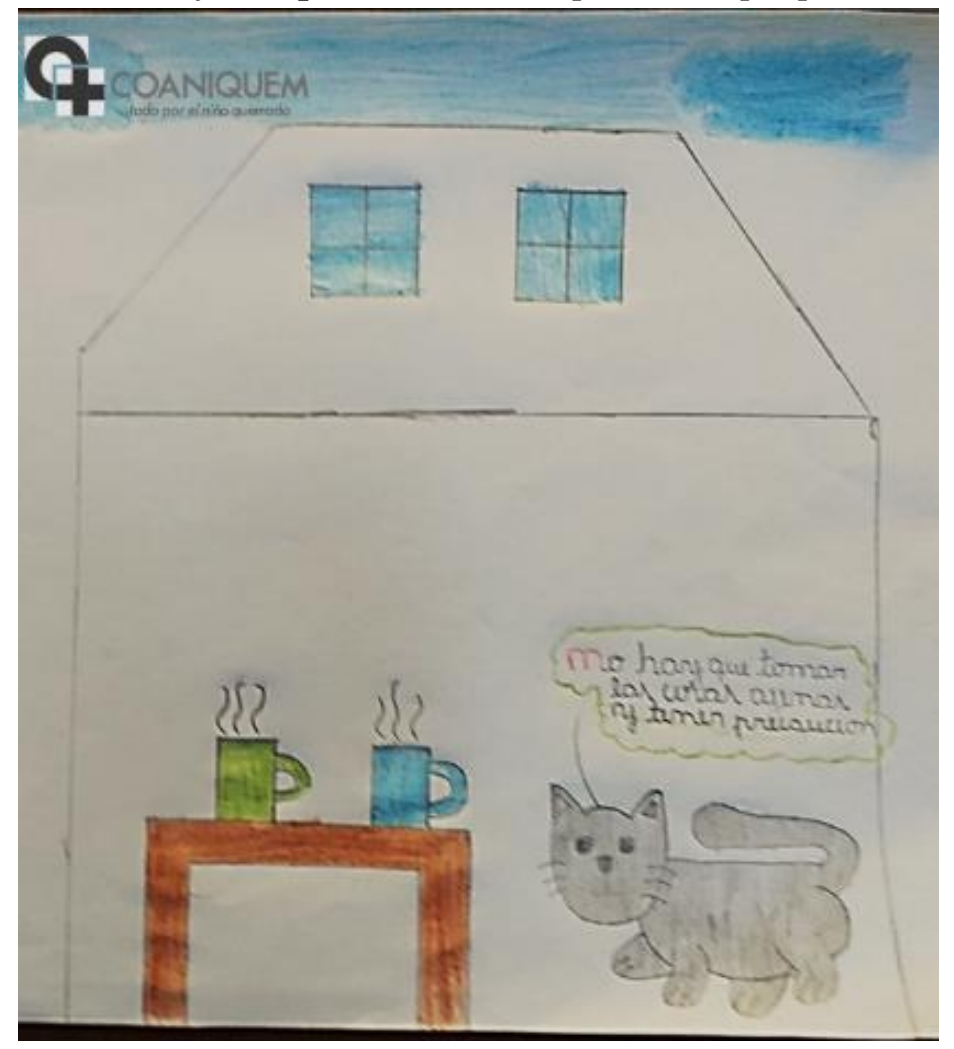

Figura 3. Dibujo demostrativo.

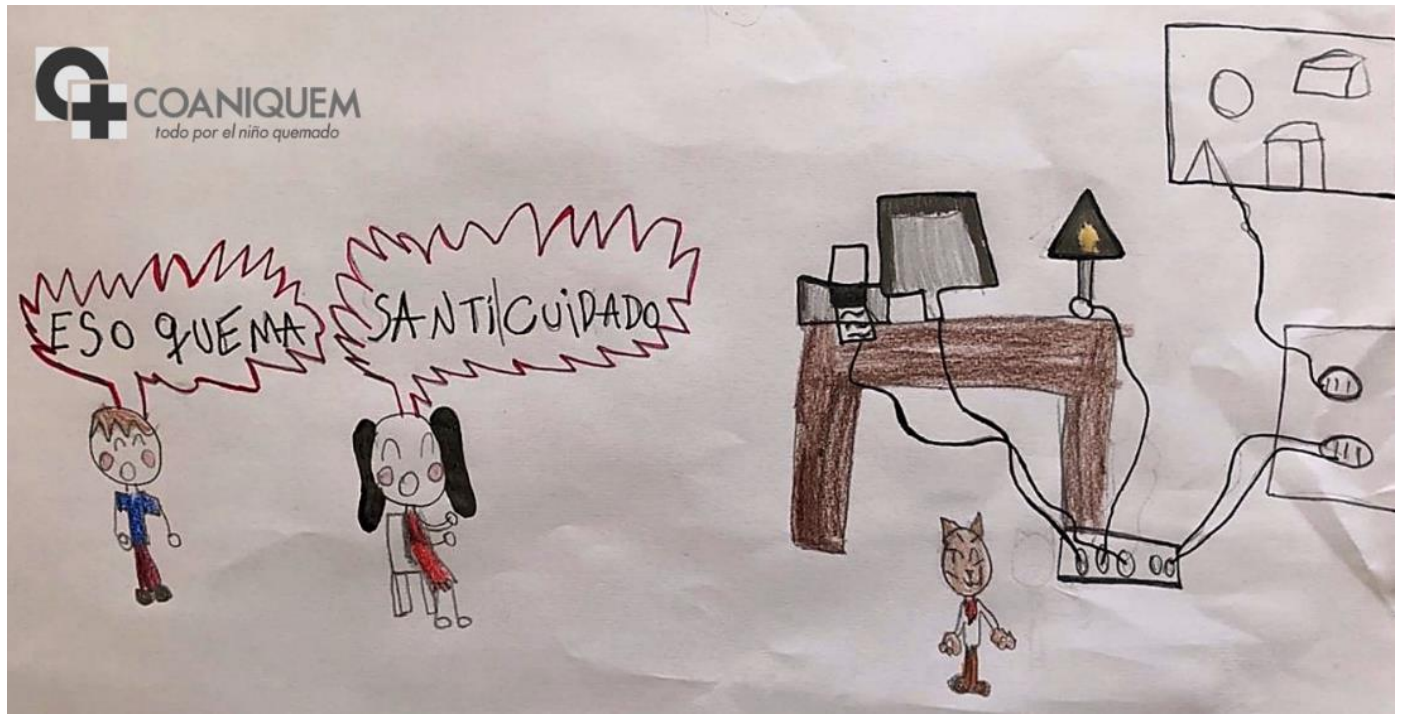


Prevención de quemaduras infantiles en tiempos de pandemia...

Figura 4. Dibujo demostrativo.

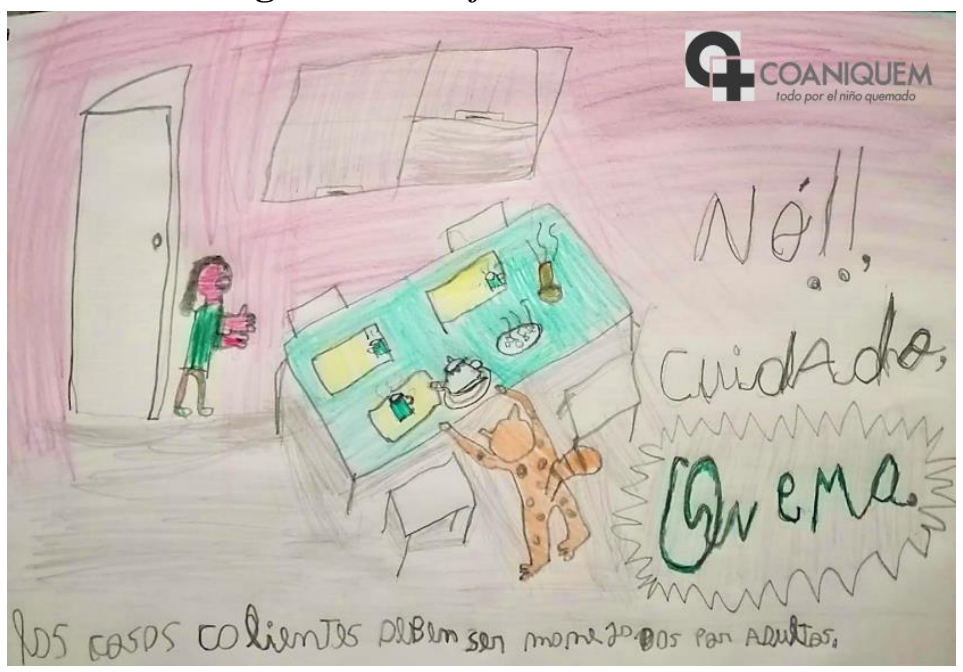

Con los dibujos recibidos se creó en cada oportunidad un video de respuesta para publicar los dibujos de los niños en redes sociales y compartir por WhatsApp. Se utilizaron los contactos y vinculación comunitaria en cada ciudad del país por medio del trabajo de las oficinas regionales de COANIQUEM, haciendo que la campaña tuviese un alcance nacional. Se publicaron videos con los dibujos seleccionados y cada uno finalizó con un consejo preventivo explicado por un profesional de COANIQUEM para reforzar el mensaje del video respectivo. El resto de los dibujos se incorporaron en un álbum de fotos en Facebook COANIQUEM y en la página web institucional. Cada vez que se publicó un video de Santi con un nuevo agente causal, la semana siguiente se publicó un video con los dibujos realizados por los niños sobre ese video. Esta modalidad alternando videos de Santi y de los dibujos se aplicó con todos los agentes causales en las semanas siguientes. Una vez establecida la campaña en Chile se contactó a las instituciones de otros países de Centro y
Sudamérica que realizan funciones similares a COANIQUEM, y con los cuales existe una vinculación histórica de trabajo conjunto en prevención y rehabilitación de quemaduras infantiles, y se les invitó a participar de la Campaña. Se hicieron los ajustes necesarios en la forma de mostrar los mecanismos, y en el lenguaje usado en la locución; e incluso, se creó un video nuevo para un mecanismo exclusivo de uno de los países que se incorporó en la campaña.

\section{Resultados de la campaña en Chile}

- 11 videos educativos de Santi, cada uno con un diferente mecanismo de quemaduras

- 165 videos de respuesta con dibujos de los niños

- 2.166 dibujos recibidos

- 126 comunas del país representadas en los dibujos recibidos

\section{Alcance internacional de la campaña}

- Participan 5 países: Bolivia (CERNIQUEM, Santa Cruz de la Sierra), Perú (ANIQUEM, Lima), Honduras (CRI- 
SAQ), Costa Rica (ASOQUEM) y Colombia (Piel para Renacer)

- 20 videos educativos de Santi, uno específico para mecanismo propio de quemaduras

- 15 videos de respuesta con dibujos de los niños

- 317 dibujos recibidos.

Algunos de los videos pueden ser revisados en el siguiente enlace:

shorturl.at/hASX0

\section{CONCLUSIONES}

La realización de una campaña virtual a través de redes sociales se presenta como una manera de acceder a la población para entregar contenidos educativos en salud, en este caso dirigida a niños y con material creado específicamente para ellos.

La campaña es interactiva ya que considera la participación activa de los destinatarios -en este caso niños- quienes no solo reciben un mensaje, sino que además tienen la posibilidad de responder al mensaje, plasmando en el dibujo su visión personal de lo percibido y pueden compartirlo. El niño a través de los dibujos expresa lo que ha interpretado e incorporado sobre la realidad percibida en el mensaje, otorgando de esta manera una confirmación de que los mensajes preventivos han sido recibidos. A partir del apoyo de los padres y profesores que facilitaban el acceso de los niños a estos videos y luego enviaban los dibujos realizados a COANIQUEM, se pudo establecer una comunicación continua con la población objetivo.

Las dificultades para el desarrollo de la campaña fueron mínimas y se
Domic Cárdenas C, Paredes Mayor C relacionaron principalmente con el acceso de la población a redes sociales, y con el cumplimiento de los plazos de envío de los dibujos para alcanzar a ser incorporados en los videos de respuesta.

El alcance de la campaña medido a través de la cantidad de dibujos recibidos en respuesta a cada publicación nos confirma que el mecanismo utilizado para promoción de la salud en esta área ha sido efectivo.

\section{AGRADECIMIENTOS}

- A COANIQUEM que permitió disponer de los recursos necesarios para llevar a cabo esta campaña innovadora.

- A Piera Elgarrista y Carolina Rodríguez, por su creatividad, permanente innovación y cariño en el desarrollo del material gráfico y audiovisual.

- A Benjamín Díaz Halvorsen, por aportar su voz para la locución de los videos de la campaña y así representar a tantos niños con quemaduras.

- Al Dr. Jorge Rojas Zegers, Sr. Rodrigo Fuentes, Sr. Alan Mackern y Srta. Mónica Berg quienes revisaron esta publicación y entregaron sus aportes para un mejor documento.

\section{REFERENCIAS BIBLIOGRÁFICAS}

1. Ministerio de Salud de Chile. Guías Clínicas AUGE Gran Quemado. Bibl Minist Salud Chile [Internet]. 2016;109. Disponible en: http://www.bibliotecaminsal.cl/wp/wpcontent/uploads/2016/04/GPC-GRANQUEMADO-FINAL-18-MARZO2016_DIAGRAMADA.pdf

2. World Health Organization. Mortality among children aged 5-14 years [Internet]. 2019 [citado 2021 Ene 13]. 
Prevención de quemaduras infantiles en tiempos de pandemia...

Disponible

https://www.who.int/news-room/fact-

sheets/detail/mortality-among-

children-aged-5-14-years

3. World Health Organization, UNICEF. Children and burns [Internet]. 2008 [citado 2021 Ene 13]. Disponible en: http://www.who.int/violence_injury_pr evention/child/en/

4. Guyer B, Ma S, Grason H, Frick KD, Perry DF, Sharkey A, et al. Early Childhood Health Promotion and Its Life Course Health Consequences. Acad Pediatr [Internet]. 2009 May 1 [citado 2021 Ene 13];9(3):142-149.e71. Disponible en: http://www.academicpedsjnl.net/article /S1876285908003148/fulltext

5. Solís FF, Domic CC, Saavedra RO, González AM. Incidencia y prevalencia de las lesiones por quemaduras en menores de 20 años. Rev Chil Pediatr. 2014;85(6):674-81.

6. World Health Organization. Burns [Internet]. 2018 [citado 2021 Ene 30]. Disponible en: https://www.who.int/news-room/factsheets/detail/burns

7. Programa de las Naciones Unidas para el Medio Ambiente, Organización Mundial de la Salud. Un Entorno Sano Para Niños Sanos: Mensajes Básicos Para Actuar [Internet]. Catalogación por la Biblioteca de la OMS; 2010 [citado 2021 Ene 30]. Disponible en: https://apps.who.int/iris/bitstream/hand le/10665/44464/9789243599885_spa.p df;jsessionid=0CDDF320D2A314BA7 8DFE927CEF922CF? sequence $=1$

8. Freddy Rodríguez Torres ÁI, Enriqueta Páez Granja RI, Jacqueline Altamirano Vaca EI, Wilmer Paguay Chávez FI,
Carolina Rodríguez Alvear III J, Calero Morales SI. Nuevas perspectivas educativas orientadas a la promoción de la salud. Educ Médica Super [Internet]. 2017 [citado 2021 Ene 13];32(4). Disponible en: http://scielo.sld.cu/pdf/ems/v31n4/a251366.pdf

9. Ministerio de Salud de Chile. Departamento de Promoción de la Salud y Participación Ciudadana [Internet]. [citado 2021 Ene 30]. Disponible en: https://dipol.minsal.cl/departamentos2/promocion-de-la-salud-yparticipacion-ciudadana/

10. Camargo-Ramos CM, Pinzón-Villate GY. La promoción de la salud en la primera infancia: evolución del concepto y su aplicación en el contexto internacional y nacional. Rev la Fac Med [Internet]. 2012 Mar 1 [citado 2021 Ene 13];60(1):S62-74. Disponible en:

https://revistas.unal.edu.co/index.php/r evfacmed/article/view/35477

11. Mistry KB, Minkovitz CS, Riley AW, Johnson SB, Grason HA, Dubay LC, et al. A New Framework for Childhood Health Promotion: The Role of Policies and Programs in Building Capacity and Foundations of Early Childhood Health. Public Health. 2012;102:168896.

12. Vázquez Fernández ME, Morell Bernabé JJ, Cuervo Valdés JJ, Fernández Segura E， Garrido Torrecillas FJ, López García R, et al. La web Familia y Salud como fuente de promoción de la salud de niños, familias y adolescentes. Pediatría Atención Primaria [Internet]. 2015 
[citado 2021 Ene 13];17(66):e117-24.

Disponible

en:

https://scielo.isciii.es/scielo.php?script

=sci_arttext\&pid=S1139-

$76322015000300006 \& \operatorname{lng}=\mathrm{e} \& \& \mathrm{nrm}=\mathrm{is}$ o\&tlng=en

13. Avendaño Porras V del C. Aprender a aprender con TIC: Estudios sobre usos de tecnología educativa en el aprendizaje de los estudiantes [Internet]. Primera Ed. Editorial Universidad De La Serena; 2020 [citado 2021 Ene 30]. 7,68. Disponible en:

http://cresur.edu.mx/2019_/libros2020/
AprenderTIC.pdf

14. Arratia JII. El dibujo y la expresión oral como evidencias en el desarrollo del pensamiento de los niños preescolares. Rev Int Ciencias Soc y Humanidades, SOCIOTAM [Internet]. 2004 [citado 2021 Ene 28];XIV(2):153-72. Disponible en: https://www.redalyc.org/articulo.oa?id $=65414206$

15. Chile $\mathrm{M}$ de $\mathrm{E}$ de, Parvularia S de E. Bases Curriculares Educacion Parvularia Subsecretaría de Educación Parvularia. 2018. 